\title{
Combined effects of the Hardy potential and lower order terms in fractional Laplacian equations
}

Yingyuan $\mathrm{Mi}^{1}$, Shuibo Huang ${ }^{1 *}$ and Canyun Huang ${ }^{2}$

\section{"Correspondence:}

huangshuibo2008@163.com

'School of Mathematics and

Computer, Northwest Minzu

University, Lanzhou, People's

Republic of China

Full list of author information is

available at the end of the article

\begin{abstract}
In this paper we consider the existence and regularity of solutions to the following nonlocal Dirichlet problems:

$$
\begin{cases}(-\Delta)^{s} u-\lambda \frac{u}{|x|^{2 s}}+u^{p}=f(x), & x \in \Omega, \\ u>0, & x \in \Omega, \\ u=0, & x \in \mathbb{R}^{N} \backslash \Omega,\end{cases}
$$

where $(-\Delta)^{s}$ is the fractional Laplacian operator, $s \in(0,1), \Omega \subset \mathbb{R}^{N}$ is a bounded domain with Lipschitz boundary such that $0 \in \Omega, f$ is a nonnegative function that belongs to a suitable Lebesgue space.
\end{abstract}

Keywords: Fractional Laplacian; Hardy potential; Regularizing effect

\section{Introduction}

Recently, the fractional Laplacian has more and more applications in physics, chemistry, biology, probability and finance. The fractional Laplacian $(-\Delta)^{s}$ is a pseudo-differential operator defined by

$$
(-\Delta)^{s} u=a_{N, s} P \cdot V \cdot \int_{\mathbb{R}^{N}} \frac{u(x)-u(y)}{|x-y|^{N+2 s}} d y, \quad s \in(0,1)
$$

where P.V. stands for the Cauchy principal value and $a_{N, s}$ is a constant given by

$$
a_{N, s}=\left(\int_{\mathbb{R}^{N}} \frac{1-\cos x}{|x|^{N+2 s}}\right)^{-1}=2^{2 s-1} \pi^{-\frac{N}{2}} \frac{\Gamma\left(\frac{N+2 s}{2}\right)}{|\Gamma(-s)|}
$$

For some equivalent definitions of $(-\Delta)^{s}$, see [1-5].

The operator $(-\Delta)^{s}$ is well defined as long as $u$ belongs to the space $C_{\text {loc }}^{1,1} \cap L_{s}$, where

$$
L_{s}=\left\{u \in L_{\mathrm{loc}}^{1}: \int_{\mathbb{R}^{N}} \frac{|u(x)|}{1+|x|^{N+2 s}}<\infty\right\} .
$$

(c) The Author(s) 2018. This article is distributed under the terms of the Creative Commons Attribution 4.0 International License (http://creativecommons.org/licenses/by/4.0/), which permits unrestricted use, distribution, and reproduction in any medium, provided you give appropriate credit to the original author(s) and the source, provide a link to the Creative Commons license, and indicate if changes were made. 
In this paper, we establish existence and regularity of solutions to the following nonlocal problem:

$$
\begin{cases}(-\Delta)^{s} u-\lambda \frac{u}{|x|^{2 s}}+u^{p}=f(x), & x \in \Omega, \\ u>0, & x \in \Omega, \\ u=0, & x \in \mathbb{R}^{N} \backslash \Omega,\end{cases}
$$

where $s \in(0,1), p>0, \Omega \subset \mathbb{R}^{N}$ is a bounded domain with Lipschitz boundary such that $0 \in \Omega, f$ is a positive measurable function in $\Omega$.

Before stating our main theorem and related results, we give some notions used in this paper.

Definition 1.1 Let $s \in(0,1), \Omega \subset \mathbb{R}^{N}$, define the fractional Sobolev space

$$
H^{s}(\Omega)=\left\{u \in L^{2}(\Omega): \frac{|u(x)-u(y)|}{|x-y|^{\frac{N+2 s}{2}}} \in L^{2}(\Omega \times \Omega)\right\}
$$

and the space $H_{0}^{s}\left(\mathbb{R}^{N}\right)$, defined as

$$
H_{0}^{s}\left(\mathbb{R}^{N}\right)=\left\{u \in H^{s}\left(\mathbb{R}^{N}\right): u=0, x \in \mathbb{R}^{N} \backslash \Omega\right\}
$$

endowed with the norm

$$
\|u\|_{H_{0}^{s}(\Omega)}=\left(\int_{\mathcal{Q}} \frac{|u(x)-u(y)|^{2}}{|x-y|^{N+2 s}}\right)^{\frac{1}{2}},
$$

where $\mathcal{Q}=\mathbb{R}^{N} \times \mathbb{R}^{N} \backslash(\mathcal{C} \Omega \times \mathcal{C} \Omega)$.

The Hardy inequality plays an important role in this paper [6-8],

$$
\Lambda_{N, s} \int_{\mathbb{R}^{N}} \frac{u^{2}}{|x|^{2 s}} d x \leq \frac{a_{N, s}}{2} \int_{\mathbb{R}^{N}} \int_{\mathbb{R}^{N}} \frac{|u(x)-u(y)|^{2}}{|x-y|^{N+2 s}} d x d y, \quad u \in C_{0}^{\infty}\left(\mathbb{R}^{N}\right),
$$

where

$$
\Lambda_{N, s}=2^{2 s} \frac{\Gamma^{2}\left(\frac{N+2 s}{4}\right)}{\Gamma^{2}\left(\frac{N-2 s}{4}\right)}
$$

is optimal and not attained.

We need to make precise the sense of solutions that we will handle here and distinguish two types of solutions, according to the regularity of $f$.

Definition 1.2 Assume $0<\lambda<\Lambda_{N, s}$. For $f \in H^{-s}(\Omega)$ we say that $u \in H_{0}^{s}(\Omega)$ is a finite energy solution to problem (1.1) if, for any $w \in H_{0}^{s}(\Omega)$,

$$
\frac{a_{N, s}}{2}\left\langle(-\Delta)^{s} u, w\right\rangle-\lambda \int_{\Omega} \frac{u w}{|x|^{2 s}} d x+\int_{\Omega} u^{p} w d x=\langle f, w\rangle,
$$


where $\langle\cdot, \cdot\rangle$ is the natural duality product between $H_{0}^{s}$ and $H^{-s}$, be defined as

$$
\left\langle(-\Delta)^{s} u, w\right\rangle_{H_{0}^{s}\left(\mathbb{R}^{N}\right)}:=P . V \cdot \int_{\mathbb{R}^{N}} \int_{\mathbb{R}^{N}} \frac{(u(x)-u(y))(w(x)-w(y))}{|x-y|^{N+2 s}} d x d y .
$$

Definition 1.3 For $f \in L^{m}(\Omega), m \geq 1$, we say that $u \in L^{1}(\Omega)$ is a weak solution to problem (1.1) if $u^{p} \in L^{1}(\Omega), u=0$ in $\mathbb{R}^{N} \backslash \Omega$ and the following equality holds:

$$
\int_{\Omega} u(-\Delta)^{s} \phi d x-\lambda \int_{\Omega} \frac{u}{|x|^{2 s}} \phi d x+\int_{\Omega} u^{p} \phi d x=\int_{\Omega} f \phi d x, \quad \forall \phi \in H_{0}^{s}(\Omega) .
$$

Recently a great attention has been devoted to understanding the role of the Hardy potential in the solvability of fractional elliptic problem; see for instance [9-13] and the references therein. In particular, Abdellaoui et al. [12] obtained regularity of solutions to the following nonlocal nonlinear problem:

$$
\begin{cases}(-\Delta)^{s} u-\lambda \frac{u}{|x|^{2 s}}=f(x), & x \in \Omega, \\ u>0, & x \in \Omega, \\ u=0, & x \in \mathbb{R}^{N} \backslash \Omega,\end{cases}
$$

with $f \in L^{m}(\Omega)$ and $0<\lambda<\Lambda_{N, s}$, where $\Lambda_{N, s}$ appears in the Hardy inequality (1.2). The main results of [12] can be summarized as follows:

- If $f \in L^{m}(\Omega), m>\frac{N}{2 s}$, the unique energy solution $u \in H_{0}^{s}(\Omega)$ to problem (1.3) with $\lambda \leq \Lambda_{N, s}$ satisfies $u \leq C|x|^{-\gamma}$ for some constants $C$ and $\gamma$.

- If $\frac{2 N}{N+2 s} \leq m<\frac{N}{2 s}$, the unique energy solution $u$ to problem (1.3) verifies $u \in L^{m_{s}^{* *}}(\Omega)$, $m_{s}^{* *}=\frac{m N}{N-2 m s}$, provided $\lambda<\Lambda_{N, s} \frac{4 N(m-1)(N-2 m s)}{m^{2}(N-2 m s)^{2}}$.

- If $1<m<\frac{2 N}{N+2 s}$, the unique weak solution $u$ to problem (1.3) verifies $u \in L^{m_{s}^{* *}}(\Omega) \cap W_{0}^{s_{1}, m_{s}^{*}}(\Omega)$ for all $s_{1}<s$ and $m_{s}^{*}=\frac{m N}{N-m s}$, provided $\lambda<\Lambda_{N, s} \frac{4 N(m-1)(N-2 m s)}{m^{2}(N-2 m s)^{2}}$.

The main objective of this work is to explain the combined influence of the Hardy potential and lower order terms on the existence and regularity of solutions to problem (1.1). The influence of the Hardy potential for fractional Laplacian was studied in [12], the main effect of the Hardy potential in (1.3) is that the weak solutions to problem (1.3) satisfy $u(x) \geq C|x|^{-\gamma}$ for some constants $C$ and $\gamma$, this fact shows that $u(x)$ is unbounded in a neighborhood of the origin, instead of $u(x) \in L^{\infty}(\Omega)$. On the other hand, it is well known that the lower order term $u^{p}$ produces a regularizing effect; see [14-17] and the references therein. Therefore, thanks to the regularizing properties of the lower order term, we will prove that summability of finite energy the solution to problem (1.1) increases as the power of the lower order term increases; see (1.4) below.

According to such a definition, we can now state our existence results for problem (1.1).

Theorem 1.4 Assume $\lambda \leq \Lambda_{N, s}$. Then, for any $f \in L^{m}(\Omega)$ with $1 \leq m \leq 1+\frac{1}{p}$, problem (1.1) has a weak solution. More precisely, $u \in H_{0}^{s}(\Omega) \cap L^{p+1}(\Omega)$.

In the case where $f \in L^{m}(\Omega)$ with $m>1+\frac{1}{p}$, we will prove the following existence result.

Theorem 1.5 Let $f \in L^{m}(\Omega)$ with $m>1+\frac{1}{p}$, and

$$
\lambda \leq \Lambda_{N, s} \frac{4 p(m-1)}{(p(m-1)+1)^{2}} .
$$


Then there exists a finite energy solution $u$ to problem (1.1) that verifies

$$
\|u\|_{L^{m_{s, p}(\Omega)}} \leq C\|f\|_{L^{m}(\Omega)}
$$

where

$$
m_{s, p}=\frac{N(p(m-1)+1)}{N-2 s} .
$$

Remark 1.6 Obviously,

$$
\frac{N(p(m-1)+1)}{N-2 s} \rightarrow+\infty \quad \text { as } p \rightarrow \infty .
$$

Thus the summability of the solution to problem (1.1) increases as $p$ increases.

Remark 1.7 When $s=1$, the above theorem was proved by Adimurthi et al. [18].

The paper is organized as follows. In Sect. 2 we collect some useful tools, such as Sobolev's imbedding theorem and a certain algebraic inequality. Furthermore, we also obtain a prior estimate of the absorption term $u^{p}$ by analyzing the associated approximating problems. The proofs of Theorem 1.4 and 1.5 will be given in Sect. 3.

\section{Useful tools and preliminaries}

In this paper, we will use the classical truncating method. Given $u$ a measurable function we consider the $k$-truncation of $u$ defined by

$$
T_{k}(u)= \begin{cases}u, & |u| \leq k \\ k \frac{u}{|u|}, & |u|>k .\end{cases}
$$

The remainder of the truncation $T_{k}(u)$ is defined as $G_{k}(u)=u-T_{k}(u)$.

We will also need the classical Sobolev theorem; for an elementary proof of this inequality, see [1].

Lemma 2.1 Let $s \in(0,1)$ and $N>2 s$. There exists a constant $C(N, s)$ such that for any measurable and compactly supported function $f: \mathbb{R}^{N} \rightarrow \mathbb{R}$,

$$
\|f\|_{\left.L^{2 *} \mathbb{R}^{N}\right)} \leq C(N, s) \int_{\mathbb{R}^{N}} \int_{\mathbb{R}^{N}} \frac{|f(x)-f(y)|^{2}}{|x-y|^{N+2 s}} d x d y,
$$

where $2_{s}^{*}=\frac{2 N}{N-2 s}$ is called the Sobolev critical exponent.

The next algebraic inequality will be used in our article.

Lemma 2.2 Let $s_{1}, s_{2} \geq 0$ and $a>0$. Then

$$
\left(s_{1}-s_{2}\right)\left(s_{1}^{a}-s_{2}^{a}\right) \geq \frac{4 a}{(a+1)^{2}}\left(s_{1}^{\frac{a+1}{2}}-s_{2}^{\frac{a+1}{2}}\right)^{2} .
$$

Proof The complete proof is given in [12], for the reader's convenience, we include here a sketch of the proof. 
If $s_{1}=0$ or $s_{2}=0$, This conclusion is obvious. We can assume $s_{1}>s_{2}>0$, let $x:=s_{2} / s_{1}$, then (2.2) is equivalent to

$$
(1-x)\left(1-x^{a}\right) \geq \frac{4 a}{(a+1)^{2}}\left(1-x^{\frac{a+1}{2}}\right)^{2}, \quad x \in(0,1) .
$$

Set

$$
h(x):=(1-x)\left(1-x^{a}\right)(a+1)^{2}-4 a\left(1-x^{\frac{a+1}{2}}\right)^{2}
$$

Rewrite $h$ as

$$
h(x):=(a-1)^{2}\left(1-x^{\frac{a+1}{2}}\right)^{2}-(a+1)^{2}\left(x^{\frac{1}{2}}-x^{\frac{a}{2}}\right)^{2} .
$$

For $a>1$, we claim that

$$
(a-1)\left(1-x^{\frac{a+1}{2}}\right) \geq(a+1)\left(x^{\frac{1}{2}}-x^{\frac{a}{2}}\right)
$$

Define

$$
h_{1}(x):=(a-1)\left(1-x^{\frac{a+1}{2}}\right)-(a+1)\left(x^{\frac{1}{2}}-x^{\frac{a}{2}}\right) .
$$

Clearly,

$$
h_{1}^{\prime}(x)=\frac{a+1}{2}\left(-(a-1) x^{\frac{a-1}{2}}-x^{\frac{1}{2}}+a x^{\frac{a-2}{2}}\right) .
$$

Thus $h_{1}^{\prime}(x) \leq 0$, here the following Young inequality will be used:

$$
x^{\frac{a}{2}-1} \leq \frac{a-1}{a} x^{\frac{a-1}{2}}+\frac{1}{a} x^{-\frac{1}{2}} .
$$

Therefore $h_{1}(x) \geq h_{1}(1)=0$, which shows that (2.3) holds.

For $a<1$. Firstly we show that

$$
(1-a)\left(1-x^{\frac{a+1}{2}}\right) \geq(a+1)\left(x^{\frac{a}{2}}-x^{\frac{1}{2}}\right)
$$

In order to do this, define

$$
h_{2}(x):=(1-a)\left(1-x^{\frac{a+1}{2}}\right)-(a+1)\left(x^{\frac{a}{2}}-x^{\frac{1}{2}}\right) .
$$

By Young's inequality, we obtain $h_{2}^{\prime}(x) \leq 0$ for all $x \in(0,1)$ and hence $h_{2}(x) \geq h_{1}(1)=0$, which shows that (2.3) holds again. Therefore, (2.2) holds.

Now we consider the following approximation problems:

$$
\begin{cases}(-\Delta)^{s} u_{n}-\lambda \frac{u_{n}}{|x|^{2 s}+\frac{1}{n}}+u_{n}^{p}=f_{n}(x), & x \in \Omega, \\ u_{n}>0, & x \in \Omega, \\ u_{n}=0, & x \in \mathbb{R}^{N} \backslash \Omega,\end{cases}
$$

where $f_{n}(x)=\frac{f(x)}{1+\frac{1}{n}}$. 
Lemma 2.3 Let $f \in L^{m}(\Omega), m \geq 1$. Then, for every $n \in \mathbb{R}$, there exists a solution $u_{n} \in H_{0}^{s}(\Omega)$ to problem (2.4) such that

$$
\int_{\Omega} u_{n}^{p m} \leq \int_{\Omega} f^{m}
$$

Proof To show estimate (2.5), we will consider the case $m>1$ and $m=1$ separately.

Case $m>1$. Choose $\phi=u_{n}^{p(m-1)}$ as a test function in (2.4), we get

$$
\begin{aligned}
& \frac{a_{N, s}}{2} \int_{\Omega} \int_{\Omega} \frac{\left(u_{n}(x)-u_{n}(y)\right)\left(u_{n}^{p(m-1)}(x)-u_{n}^{p(m-1)}(y)\right)}{|x-y|^{N+2 s}}+\int_{\Omega} u_{n}^{p m} \\
& \quad=\int_{\Omega} f u_{n}^{p(m-1)}+\lambda \int_{\Omega} \frac{u_{n}^{p(m-1)+1}}{|x|^{2 s}} .
\end{aligned}
$$

Then (1.2) and (2.2) yield

$$
\begin{aligned}
& \frac{a_{N, s}}{2}\left(\frac{4 p(m-1)}{(p(m-1)+1)^{2}}-\frac{\lambda}{\Lambda_{N, s}}\right) \int_{\Omega} \int_{\Omega} \frac{\left(u_{n}^{\frac{p(m-1)+1}{2}}(x)-u_{n}^{\frac{p(m-1)+1}{2}}(y)\right)^{2}}{|x-y|^{N+2 s}} \\
& \quad+\int_{\Omega} u_{n}^{p m} \leq \int_{\Omega} f u_{n}^{p(m-1)} .
\end{aligned}
$$

Therefore

$$
\int_{\Omega} u_{n}^{p m} \leq \int_{\Omega} f u_{n}^{p(m-1)}
$$

provided

$$
\lambda \leq \frac{4 p(m-1) \Lambda_{N, s}}{(p(m-1)+1)^{2}} .
$$

Applying Hölder's inequality on the right-hand-side of (2.6), we obtain

$$
\int_{\Omega} f u_{n}^{p(m-1)} \leq\|f\|_{L^{m}(\Omega)}\left(\int_{\Omega} u_{n}^{p m}\right)^{\frac{1}{m^{\prime}}},
$$

where $m^{\prime}=\frac{m}{m-1}$, which together with (2.6), implies that (2.5) holds.

Case $m=1$. Using $\frac{T_{k}\left(u_{n}\right)}{k}$ as a test function in (2.4), we get

$$
\begin{aligned}
& \frac{a_{N, s}}{2 k} \int_{\Omega} \int_{\Omega} \frac{\left(u_{n}(x)-u_{n}(y)\right)\left(T_{k}\left(u_{n}(x)\right)-T_{k}\left(u_{n}(y)\right)\right)}{|x-y|^{N+2 s}} \\
& -\frac{\lambda}{k} \int_{\Omega} \frac{u_{n} T_{k}\left(u_{n}\right)}{|x|^{2 s}}+\int_{\Omega} u_{n}^{p} \frac{T_{k}\left(u_{n}\right)}{k}=\int_{\Omega} f_{n} \frac{T_{k}\left(u_{n}\right)}{k} .
\end{aligned}
$$

Since for any $\sigma \in \mathbb{R}^{N}, \sigma=T_{k}(\sigma)+G_{k}(\sigma)$,

$$
\begin{aligned}
& \left(u_{n}(x)-u_{n}(y)\right)\left(T_{k}\left(u_{n}(x)\right)-T_{k}\left(u_{n}(y)\right)\right) \\
& \quad=\left(T_{k}\left(u_{n}(x)\right)-T_{k}\left(u_{n}(y)\right)\right)^{2}+\left(T_{k}\left(u_{n}(x)\right)-T_{k}\left(u_{n}(y)\right)\right)\left(G_{k}\left(u_{n}(x)\right)-G_{k}\left(u_{n}(y)\right)\right) .
\end{aligned}
$$


Moreover, by Lemma 4 in [19], we know that

$$
\left(T_{k}\left(u_{n}(x)\right)-T_{k}\left(u_{n}(y)\right)\right)\left(G_{k}\left(u_{n}(x)\right)-G_{k}\left(u_{n}(y)\right)\right) \geq 0,
$$

and using Hardy's inequality (1.2), we get

$$
\frac{a_{N, s}}{2 k}\left(1-\frac{\lambda}{\Lambda_{N, s}}\right) \int_{\Omega} \int_{\Omega} \frac{\left(T_{k}\left(u_{n}(x)\right)-T_{k}\left(u_{n}(y)\right)\right)^{2}}{|x-y|^{N+2 s}}+\int_{\Omega} u_{n}^{p} \frac{T_{k}\left(u_{n}\right)}{k} \leq \int_{\Omega} f_{n} \frac{T_{k}\left(u_{n}\right)}{k} .
$$

Since $\lambda<\Lambda_{N, s}$, we have

$$
\int_{\Omega} u_{n}^{p} \frac{T_{k}\left(u_{n}\right)}{k} \leq \int_{\Omega} f \frac{T_{k}\left(u_{n}\right)}{k} \leq \int_{\Omega} f
$$

Fatou's lemma implies, for $k \rightarrow \infty$, that estimate (2.5) holds.

\section{Proof of main results}

Let us begin with the proof of Theorem 1.4.

Proof of Theorem 1.4 Set $f_{n}=\frac{f}{1+\frac{1}{n}}$, obviously, $f_{n} \rightarrow f$ in $L^{1}(\Omega)$ as $n \rightarrow \infty$. Let $\phi=T_{k}\left(u_{n}\right)$ as a test function in (2.4), we have

$$
\begin{aligned}
& \frac{a_{N, s}}{2} \int_{\Omega} \int_{\Omega} \frac{\left(u_{n}(x)-u_{n}(y)\right)\left(T_{k}\left(u_{n}(x)\right)-T_{k}\left(u_{n}(y)\right)\right)}{|x-y|^{N+2 s}} \\
& -\lambda \int_{\Omega} \frac{u_{n} T_{k}\left(u_{n}\right)}{|x|^{2 s}+\frac{1}{n}}+\int_{\Omega} u_{n}^{p} T_{k}\left(u_{n}\right)=\int_{\Omega} f_{n} T_{k}\left(u_{n}\right) .
\end{aligned}
$$

Since for any $\sigma \in \mathbb{R}, \sigma=T_{k}(\sigma)+G_{k}(\sigma)$,

$$
\begin{aligned}
& \left(u_{n}(x)-u_{n}(y)\right)\left(T_{k}\left(u_{n}(x)\right)-T_{k}\left(u_{n}(y)\right)\right) \\
& =\left(T_{k}\left(u_{n}(x)\right)-T_{k}\left(u_{n}(y)\right)\right)^{2} \\
& \quad+\left(T_{k}\left(u_{n}(x)\right)-T_{k}\left(u_{n}(y)\right)\right)\left(G_{k}\left(u_{n}(x)\right)-G_{k}\left(u_{n}(x)\right)\right) .
\end{aligned}
$$

Moreover, by Lemma 4 in [19], we know that

$$
\left(T_{k}\left(u_{n}(x)\right)-T_{k}\left(u_{n}(y)\right)\right)\left(G_{k}\left(u_{n}(x)\right)-G_{k}\left(u_{n}(y)\right)\right) \geq 0 .
$$

Therefore, (3.1)-(3.2) lead to

$$
\frac{a_{N, s}}{2} \int_{\Omega} \int_{\Omega} \frac{\left(T_{k}\left(u_{n}(x)\right)-T_{k}\left(u_{n}(y)\right)\right)^{2}}{|x-y|^{N+2 s}}+\int_{\Omega} u_{n}^{p} T_{k}\left(u_{n}\right) \leq k \int_{\Omega}|f|+\lambda \int_{\Omega} \frac{u_{n}^{2}}{|x|^{2 s}} .
$$

Recall that

$$
\begin{aligned}
\int_{\Omega} \frac{u_{n}^{2}}{|x|^{2 s}} & =\int_{\Omega} \frac{\left(T_{k}\left(u_{n}\right)+G_{k}\left(u_{n}\right)\right)^{2}}{|x|^{2 s}} \\
& =\int_{\Omega} \frac{T_{k}^{2}\left(u_{n}\right)}{|x|^{2 s}}+\int_{\Omega} \frac{G_{k}^{2}\left(u_{n}\right)}{|x|^{2 s}}+2 \int_{\Omega} \frac{T_{k}\left(u_{n}\right) G_{k}\left(u_{n}\right)}{|x|^{2 s}} .
\end{aligned}
$$


On the other hand, using $G_{k}\left(u_{n}\right)$ as a test function in (2.4), we have

$$
\begin{aligned}
& \frac{a_{N, s}}{2} \int_{\Omega} \int_{\Omega} \frac{\left|G_{k} u_{n}(x)-G_{k} u_{n}(x)\right|^{2}}{|x-y|^{N+2 s}}-\lambda \int_{\Omega} \frac{u_{n} G_{k}\left(u_{n}\right)}{|x|^{2 s}} \\
& \quad+\int_{\Omega} u_{n}^{p} G_{k}\left(u_{n}\right) \leq \int_{\Omega} f G_{k}\left(u_{n}\right) .
\end{aligned}
$$

Moreover, $u_{n} G_{k}\left(u_{n}\right)=G_{k}^{2}\left(u_{n}\right)+T_{k}\left(u_{n}\right) G_{k}\left(u_{n}\right)$, thus this fact combined with (3.6), implies that

$$
\begin{aligned}
& \frac{a_{N, s}}{2} \int_{\Omega} \int_{\Omega} \frac{\left|G_{k} u_{n}(x)-G_{k} u_{n}(x)\right|^{2}}{|x-y|^{N+2 s}}-\lambda \int_{\Omega} \frac{G_{k}^{2}\left(u_{n}\right)}{|x|^{2 s}}+\int_{\Omega} u_{n}^{p} G_{k}\left(u_{n}\right) \\
& \quad \leq k \int_{\Omega} f_{n}+k \lambda \int_{\Omega} \frac{G_{k}\left(u_{n}\right)}{|x|^{2 s}} .
\end{aligned}
$$

Applying the Young inequality on the right-hand-side of (3.7), we get

$$
\int_{\Omega} \frac{G_{k}\left(u_{n}\right)}{|x|^{2 s}} \leq \frac{1}{2} \int_{\Omega} \frac{G_{k}^{2}\left(u_{n}\right)}{|x|^{2 s}}+\frac{1}{2} \int_{\Omega} \frac{1}{|x|^{2 s}}
$$

Taking into account that $\lambda<\Lambda_{N, s}$, by the Hardy inequality we obtain

$$
\int_{\Omega} \int_{\Omega} \frac{\left|G_{k}\left(u_{n}(x)\right)-G_{k}\left(u_{n}(y)\right)\right|^{2}}{|x-y|^{N+2 s}} \leq C\left(f, k, \lambda, \Lambda_{N, s}\right) .
$$

Therefore $\left\{G_{k}\left(u_{n}\right)\right\}_{n \in \mathbb{R}}$ is uniformly bounded in $H_{0}^{s}(\Omega)$, it implies

$$
\int_{\Omega} \frac{G_{k}^{2}\left(u_{n}\right)}{|x|^{2 s}} \leq C\left(f, k, \lambda, \Lambda_{N, s}\right)
$$

Then we get

$$
\begin{aligned}
\int_{\Omega} \frac{u_{n}^{2}}{|x|^{2 s}} & =\int_{\Omega} \frac{\left(T_{k}\left(u_{n}\right)+G_{k}\left(u_{n}\right)\right)^{2}}{|x|^{2 s}} \\
& =\int_{\Omega} \frac{T_{k}^{2}\left(u_{n}\right)}{|x|^{2 s}}+\int_{\Omega} \frac{G_{k}^{2}\left(u_{n}\right)}{|x|^{2 s}}+2 \int_{\Omega} \frac{T_{k}\left(u_{n}\right) G_{k}\left(u_{n}\right)}{|x|^{2 s}} \\
& \leq C\left(f, k, \lambda, \Lambda_{N, s}\right) .
\end{aligned}
$$

Putting together (3.4)-(3.5) and (3.8), it follows that

$$
\frac{a_{N, s}}{2} \int_{\Omega} \int_{\Omega} \frac{\left(T_{k}\left(u_{n}(x)\right)-T_{k}\left(u_{n}(y)\right)\right)^{2}}{|x-y|^{N+2 s}}+\int_{\Omega} T_{k}^{p+1}\left(u_{n}\right) \leq C\left(f, k, \lambda, \Lambda_{N, s}\right)
$$

We deduce that $T_{k}\left(u_{n}\right)$ is uniformly bounded in $H_{0}^{s}(\Omega) \cap L^{p+1}(\Omega)$. Then we pass to the limit in the approximation problem (2.4); up to a subsequence, there exists a function $u \in H_{0}^{s}(\Omega) \cap L^{p+1}(\Omega)$. 
Now we want to prove that $u_{n}^{p} \rightarrow u$ in $L^{1}(\Omega)$. Let $\psi_{i}(\sigma)$ be defined by

$$
\psi_{i}(\sigma)= \begin{cases}1, & \sigma \geq t \\ 0, & |\sigma|<t \\ -1, & \sigma \leq-t\end{cases}
$$

Choosing $\phi=\psi_{i}\left(u_{n}\right)$ as a test function in (2.4), we get

$$
\int_{\Omega} u_{n}^{p} \psi_{i}\left(u_{n}\right) \leq \int_{\Omega} f \psi_{i}\left(u_{n}\right)
$$

which implies that

$$
\int_{\left\{u_{n}>t\right\} \cap \Omega} u_{n}^{p} \leq \int_{\left\{u_{n}>t\right\} \cap \Omega} f .
$$

Let $E$ is any measurable subset of $\Omega$. For any $t>0$ we have

$$
\int_{E} u_{n}^{p} \leq t^{p}|E|+\int_{E \cap\left\{u_{n}>t\right\}} u_{n}^{p} \leq t^{p}|E|+\int_{\left\{u_{n}>t\right\}}|f| .
$$

The above fact and $f \in L^{1}(\Omega)$ allow us to say that, for any given $\varepsilon>0$, there exists $t_{\varepsilon}$ such that

$$
\int_{\left\{u_{n}>t_{\varepsilon}\right\}}|f| \leq \varepsilon
$$

Hence

$$
\int_{E} u_{n}^{p} \leq t_{\varepsilon}^{p}|E|+\varepsilon
$$

Therefore

$$
\lim _{|E| \rightarrow 0} \int_{E} u_{n}^{p} \leq \varepsilon .
$$

Thus we prove that $\lim _{|E| \rightarrow 0} \int_{E} u_{n}^{p}=0$. Vitali's theorem implies that $u_{n}^{p} \rightarrow u^{p}$ in $L^{1}(\Omega)$ i.e.

$$
\lim _{n \rightarrow \infty} \int_{\Omega} u_{n}^{p}=\int_{\Omega} u^{p}
$$

Let us show Theorem 1.5, that is, the existence of solution to problem (1.1) in the case where $f \in L^{m}(\Omega)$ with $m>\frac{p+1}{p}$.

Proof of Theorem 1.5 Define $\beta=p(m-1)$, that satisfies $p+\beta=\beta m^{\prime}$. Using $\phi=u_{n}^{\beta}$ as a test function in (2.4), we have

$$
\frac{a_{N, s}}{2} \int_{\Omega} \int_{\Omega} \frac{\left(u_{n}(x)-u_{n}(y)\right)\left(u_{n}^{\beta}(x)-u_{n}^{\beta}(y)\right)}{|x-y|^{N+2 s}}+\int_{\Omega} u_{n}^{p+\beta}=\int_{\Omega} f_{n} u_{n}^{\beta}+\lambda \int_{\Omega} \frac{u_{n}^{\beta+1}}{|x|^{2 s}} .
$$


Now, by Lemma 2.2, we get

$$
\left(u_{n}(x)-u_{n}(y)\right)\left(u_{n}^{\beta}(x)-u_{n}^{\beta}(y)\right) \geq \frac{4 \beta}{(\beta+1)^{2}}\left(u_{n}^{\frac{\beta+1}{2}}(x)-u_{n}^{\frac{\beta+1}{2}}(y)\right)^{2} .
$$

Using Hardy's inequality, we have

$$
\int_{\Omega} \frac{u_{n}^{\beta+1}}{|x|^{2 s}}=\int_{\Omega} \frac{\left(u_{n}^{\frac{\beta+1}{2}}\right)^{2}}{|x|^{2 s}} \leq \frac{a_{N, s}}{2 \Lambda_{N, s}} \int_{\Omega} \frac{\left(u_{n}^{\frac{\beta+1}{2}}(x)-u_{n}^{\frac{\beta+1}{2}}(y)\right)^{2}}{|x-y|^{N+2 s}} .
$$

We conclude that

$$
\begin{aligned}
& \frac{a_{N, s}}{2}\left(\frac{4 \beta}{(\beta+1)^{2}}-\frac{\lambda}{\Lambda_{N, s}}\right) \int_{\Omega} \int_{\Omega} \frac{\left(u_{n}^{\frac{\beta+1}{2}}(x)-u_{n}^{\frac{\beta+1}{2}}(y)\right)^{2}}{|x-y|^{N+2 s}}+\int_{\Omega} u_{n}^{p+\beta} \\
& \quad \leq\|f\|_{L^{m}(\Omega)}\left(\int_{\Omega} u_{n}^{\beta m^{\prime}}\right)^{\frac{1}{m^{\prime}}} .
\end{aligned}
$$

With this choice of $\beta$, by Lemma 2.3 we obtain

$$
\int_{\Omega} \int_{\Omega} \frac{\left(u_{n}^{\frac{\beta+1}{2}}(x)-u_{n}^{\frac{\beta+1}{2}}(y)\right)^{2}}{|x-y|^{N+2 s}} \leq C\|f\|_{L^{m}(\Omega)}
$$

By Lemma 2.1, we arrive at

$$
\left(\int_{\Omega} u_{n}^{\frac{(\beta+1) 2_{s}^{*}}{2}}\right)^{\frac{1}{2_{s}^{*}}} \leq C\|f\|_{L^{m}(\Omega)}
$$

Furthermore,

$$
\frac{(\beta+1) 2_{s}^{*}}{2}=\frac{N(p(m-1)+1)}{N-2 s}=m_{s, p}
$$

As a consequence there exists a function $u \in L^{\frac{(\beta+1) 2_{s}^{*}}{2}}(\Omega)$.

Finally, we want to prove that $u_{n}^{p} \rightarrow u^{p}$ in $L^{1}(\Omega)$. Using (3.10) as a test function in (2.4), we have

$$
\int_{\Omega} u_{n}^{p} \psi_{i}\left(u_{n}\right) \leq \int_{\Omega} f \psi_{i}\left(u_{n}\right)
$$

For any $t>0$ and $E \subset \Omega$ is measurable. we get

$$
\int_{E} u_{n}^{p} \leq t^{p}|E|+\int_{E \cap\left\{u_{n}>t\right\}} u_{n}^{p} \leq t^{p}|E|+\int_{\left\{u_{n}>t\right\}}|f| .
$$

There exists $t_{\varepsilon}$ such that

$$
\int_{E} u_{n}^{p} \leq t_{\varepsilon}^{p}|E|+\varepsilon
$$


We see that $|E| \rightarrow 0$ implies

$$
\int_{E} u_{n}^{p} \leq \varepsilon
$$

i.e., the sequence $u_{n}^{p}$ is equiintegrable. Consequently

$$
\lim _{n \rightarrow \infty} \int_{\Omega} u_{n}^{p}=\int_{\Omega} u^{p}
$$

Thus we have proved the existence result.

\section{Conclusion}

In this paper, we main study the regularizing effect of a nonlinear term $u^{p}$, and the influence of the Hardy potential on the existence of solutions to fractional Laplacian equations. Specifically, the positive effect of the nonlinear term $u^{p}$ is shown.

\section{Acknowledgements}

The authors would like to express their gratitude to the anonymous referee for his/her kind suggestions and helpful advices which have improved the final form of the manuscript.

\section{Funding}

This research was partially supported by the National Science Foundation of China (Nos. 11401473, 11761059), Science and Technology Planning Project of Gansu Province (No. 1610RJZA102), Fundamental Research Funds for the Central Universities (Nos. 31920170001, 31920170147) and research and innovation teams of Northwest Minzu University.

\section{Competing interests}

The authors declare to have no competing interests.

\section{Authors' contributions}

The authors contributed equally and significantly in writing this article. All authors read and approved the final manuscript.

\section{Author details}

${ }^{1}$ School of Mathematics and Computer, Northwest Minzu University, Lanzhou, People's Republic of China. ${ }^{2}$ Department of Applied Mathematics, Lanzhou University of Technology, Lanzhou, People's Republic of China.

\section{Publisher's Note}

Springer Nature remains neutral with regard to jurisdictional claims in published maps and institutional affiliations.

Received: 12 January 2018 Accepted: 16 April 2018 Published online: 24 April 2018

References

1. Di Nezza, E., Palatucci, G., Valdinoci, E.: Hitchhiker's guide to the fractional Sobolev spaces. Bull. Sci. Math. 136 521-573 (2012)

2. Bucur, C., Valdinoci, E.: Nonlocal Diffusion and Applications. Lecture Notes of the Unione Matematica Italiana, vol. 20. Springer, Cham; Unione Matematica Italiana, Bologna, xii + 155 pp. (2016)

3. Silvestre, L.: Regularity of the obstacle problem for a fractional power of the Laplace operator. Ph.D. thesis, The University of Texas at Austin, 95 pp. (2005)

4. Servadei, R., Valdinoci, E.: On the spectrum of two different fractional operators. Proc. R. Soc. Edinb., Sect. A 144 $831-855$ (2014)

5. Musina, R., Nazarov, A.: On fractional Laplacians. Commun. Partial Differ. Equ. 39, 1780-1790 (2014)

6. Abdellaoui, B., Bentifour, R.: Caffarelli-Kohn-Nirenberg type inequalities of fractional order with applications. J. Funct. Anal. 272, 3998-4029 (2017)

7. Abdellaoui, B., Peral, I., Primo, A.: A remark on the fractional Hardy inequality with a remainder term. C. R. Acad. Sci. Paris, Ser. I 352, 299-303 (2014)

8. Frank, R.L., Seiringer, R.: Non-linear ground state representations and sharp Hardy inequalities. J. Funct. Anal. 255, 3407-3430 (2008)

9. Tzirakis, K.: Sharp trace Hardy-Sobolev inequalities and fractional Hardy-Sobolev inequalities. J. Funct. Anal. 270 , 4513-4539 (2016)

10. Nguyen, V.: Some trace Hardy type inequalities and trace Hardy-Sobolev-Maz'ya type inequalities. J. Funct. Anal. 270, 4117-4151 (2016) 
11. Barrios, B., Medina, M., Peral, I.: Some remarks on the solvability of non-local elliptic problems with the Hardy potential. Commun. Contemp. Math. 16, 1350046 (2014)

12. Abdellaoui, B., Medina, M., Peral, I., Primo, A.: The effect of the Hardy potential in some Calderón-Zygmund properties for the fractional Laplacian. J. Differ. Equ. 260, 8160-8206 (2016)

13. Dipierro, S., Montoro, L., Peral, I., Sciunzi, B.: Qualitative properties of positive solutions to nonlocal critical problems involving the Hardy-Leray potential. Calc. Var. Partial Differ. Equ. 55(4), Article 99 (2016)

14. Arcoya, D., Boccardo, L.: Regularizing effect of the interplay between coefficients in some elliptic equations. J. Funct. Anal. 268, 1053-1308 (2015)

15. Arcoya, D., Boccardo, L.: Regularizing effect of $L^{9}$ interplay between coefficients in some elliptic equations. J. Math. Pures Appl. 111, 106-125 (2018)

16. Boccardo, L:: Marcinkiewicz estimates for solutions of some elliptic problems with nonregular data. Ann. Mat. Pura Appl. 188, 591-601 (2009)

17. Boccardo, L., Gallouët, T., Vázquez, J.: Nonlinear elliptic equations in $\mathbf{R}^{N}$ without growth restrictions on the data. J. Differ. Equ. 105, 334-363 (1993)

18. Adimurthi, A., Boccardo, L., Cirmi, G., Orsina, L.: The regularizing effect of lower order terms in elliptic problems involving Hardy potential. Adv. Nonlinear Stud. 17, 311-317 (2017)

19. Leonori, T., Peral, I., Primo, A., Soria, F.: Basic estimates for solutions of a class of nonlocal elliptic and parabolic equations. Discrete Contin. Dyn. Syst. 35, 6031-6068 (2015)

\section{Submit your manuscript to a SpringerOpen ${ }^{\circ}$ journal and benefit from:}

- Convenient online submission

- Rigorous peer review

- Open access: articles freely available online

- High visibility within the field

- Retaining the copyright to your article

Submit your next manuscript at $\boldsymbol{~ s p r i n g e r o p e n . c o m ~}$ 